\title{
XIII Multifrequency Behaviour of High Energy Cosmic Sources Concluding Remarks IV
}

\author{
René Hudec ${ }^{1,2,3 *}$ \\ ${ }^{1}$ Astronomical Institute of the Academy of Sciences of the Czech Republic \\ Fricova 298 - CZ 25165 Ondrejov, Czech Republic \\ ${ }^{2}$ Czech Technical University in Prague, Faculty of Electrical Engineering, \\ Technicka 2, CZ 16000 Prague, Czech Republic \\ ${ }^{3}$ Kazan Federal University, Kazan, Russian Federation \\ E-mail: rene.hudec@gmail.com
}

This paper contains the printed version of the concluding remarks I gave at the Conference XIII Multi-frequency Behaviour of High Energy Cosmic Sources Workshop, 3-8 June, 2019 in Palermo, Italy.

Multifrequency Behaviour of High Energy Cosmic Sources - XIII - MULTIF2019

3-8 June 2019

Palermo, Italy

* Speaker.

${ }^{\dagger}$ A footnote may follow. 


\section{Introduction}

This paper represents the paper version of the concluding remarks talk I gave at the Conference XIII Multifrequency Behaviour of High Energy Cosmic Sources Workshop, held 3-8 June, 2019 in Palermo, Italy.

The goal of the conference was to bring together researchers who are involved in various topics at the forefront of multifrequency astrophysics. The workshop focussed on the recent experimental and theoretical results and achievments in order to advance our understanding of various astrophysical objects based on multispectral approach.

The goals of the conference were, in my opinion, achieved by an emphasis on a synergy between data from ground-based and space-based experiments, as well as results from theoretical developments. This work at the forefront of multifrequency science is very likely to result in scientific papers of significant importance.

In my opinion, the main goal of the conference, which was to discuss in a unique and collaborative setting a broad range of topics in modern multifrequency and multispectral astrophysics, has been fully accomplished. The scope of the conference has provided a suitable framework for each participant who (while obviously not involved in all the topics discussed), was able to acquire a general view of the main experimental and theoretical results currently obtained and presented and discussed at the conference.

Last but not least, it is evident that such an up-to-date view of the current multifrequency research on various astrophysical sources, as presented by conference speakers and participants, can help guide future research projects by the participants, and surely will encourage collaborative efforts across various topical areas of multispectral research in general.

\section{Few subjective comments}

There were nice reviews presented at the conference by various key speakers, covering both theory as well as experiment. Over last years there was great progress both in theory as well as observation/experiment as illustrated by numerous speakers. New detailed observations and simulations on numerous high-energy (HE) objects were presented as well. As usual, few talks presented non-traditional and some even controversial ideas resulting in lively but still friendly discussion.

Quite common conclusion of many speakers was that we need more data, and better statistics, with focus on HE and even VHE/UHECR emissions, There is also a need for better monitoring, multi-lambda observations. Robotic Telescopes and novel space projects and experiments may help significantly, New view of old huge databases (machine learning) was also addressed, along with many other topics.

This time, to make a review is extremely difficult as the topic covered was very very wide if compared with previous conferences. In my opinion, the 2019 Frascati Workshop in Mondello, namely XIII Multifrequency Behaviour of High Energy Cosmic Sources, represented another confirmation of Franco Giovannelli's "Vulcano Theorem" (more details are given in Professor Franco Giovannelli's concluding talk). Apart from this theorem, the keys for a productive workshop are, in my opinion, very obvious: a nice environment, especially delicious dining, and staying together. 
This allows contacts and discussions among participants not only during the scientific sessions, but for the whole duration of the conference.

In comparing the previous Frascati Vulcano/Mondello workshops with the 2019 conference, it appears to me that this was innovative in the sense that it covered again a wider range of scientific fields than before, even while focusing on the multispectral astrophysics. Continuation in including a wider range of topics can be considered as a positive move. For example in X-ray astronomy and astrophysics, there are communities working in various aspects, and bringing them together with the galactic and extragalactic X-ray astronomy community is surely beneficial for all. And another important factor was the growing participation of young and woman researchers.

And what is also very important, there has been a strong participation both of leaders as well as young scientists in these fields. Most importantly, there have been many occasions for young researchers to meet famous scientists they had known previously only from the literature, both in the question periods and in informal settings.

Last but not least, I note the very high quality of the invited and review talks - this is not trivial as we face the problem of the declining quality of such talks at numerous other conferences.

In my opinion, the following selected pictures taken during the conference will better illustrate the scope, atmosphere, and success of the conference XIII Multifrequency Behaviour of High Energy Cosmic Sources better than any more words.

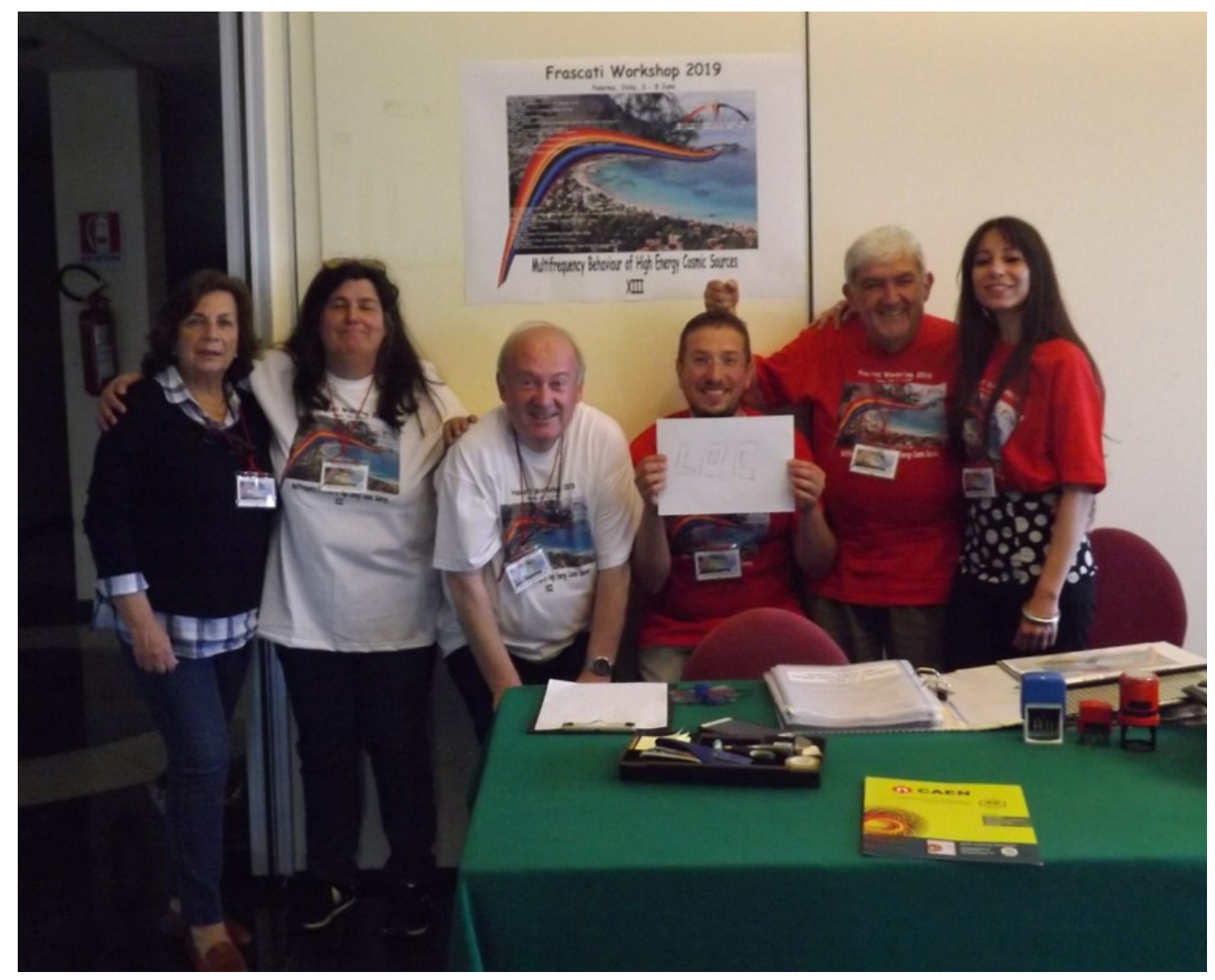

Figure 1: LOC of the conference. 


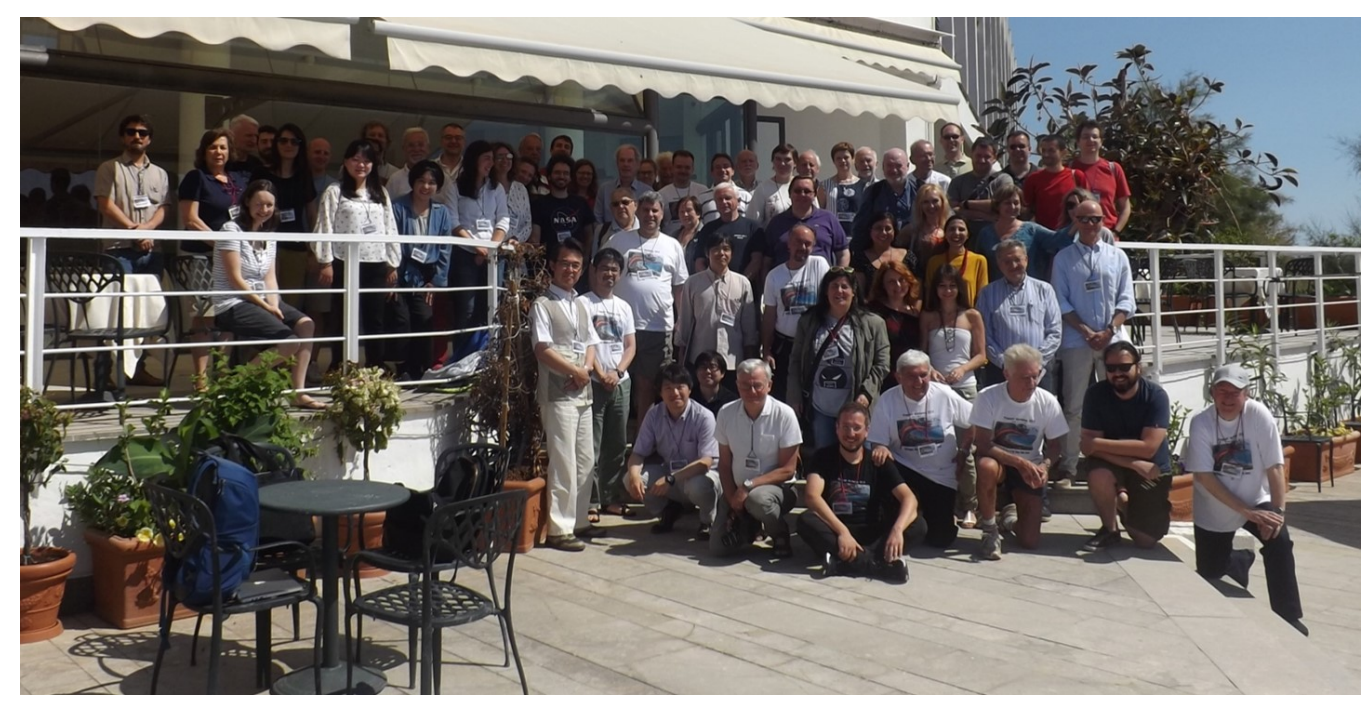

Figure 2: Conference group photograph.

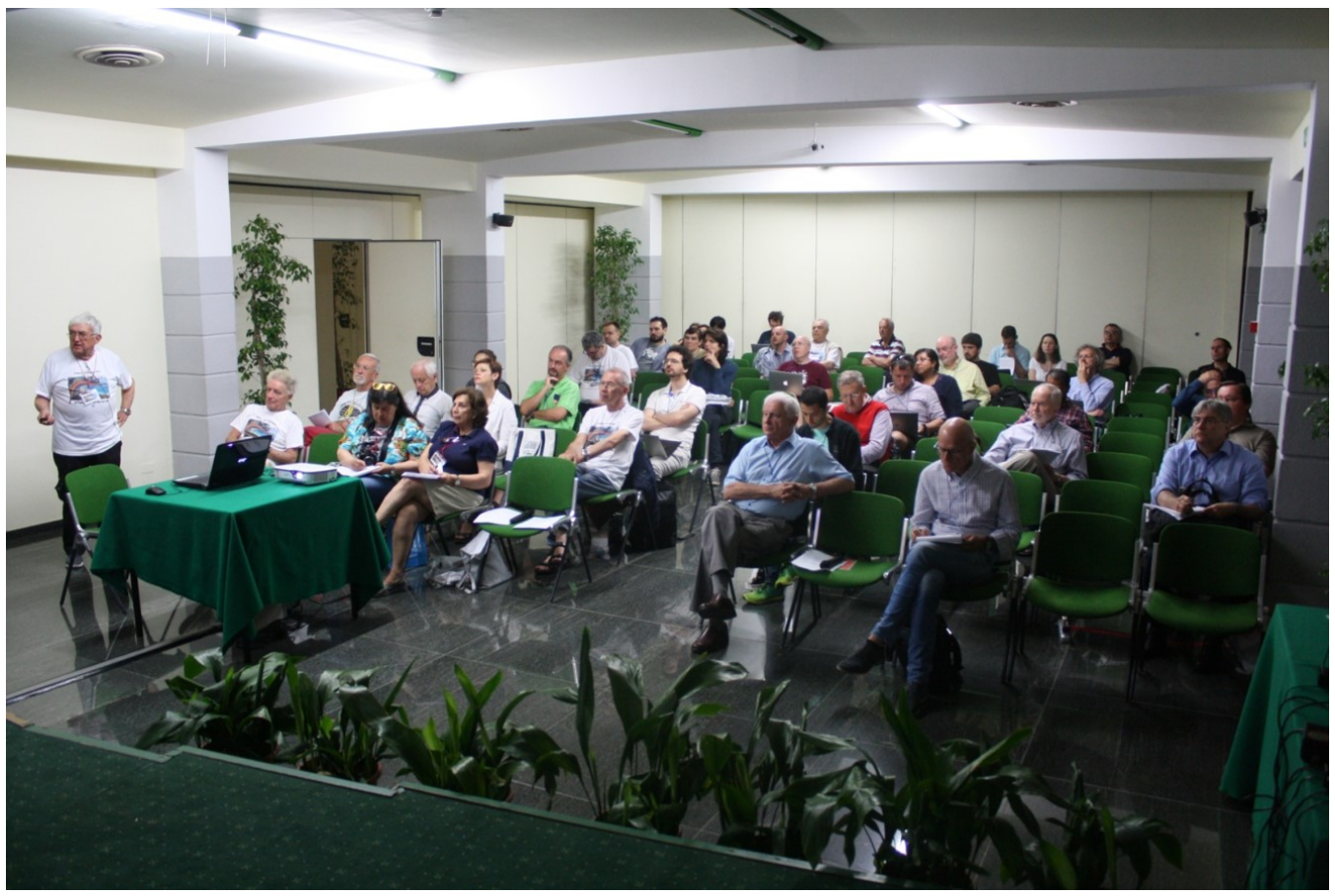

Figure 3: Conference session. 


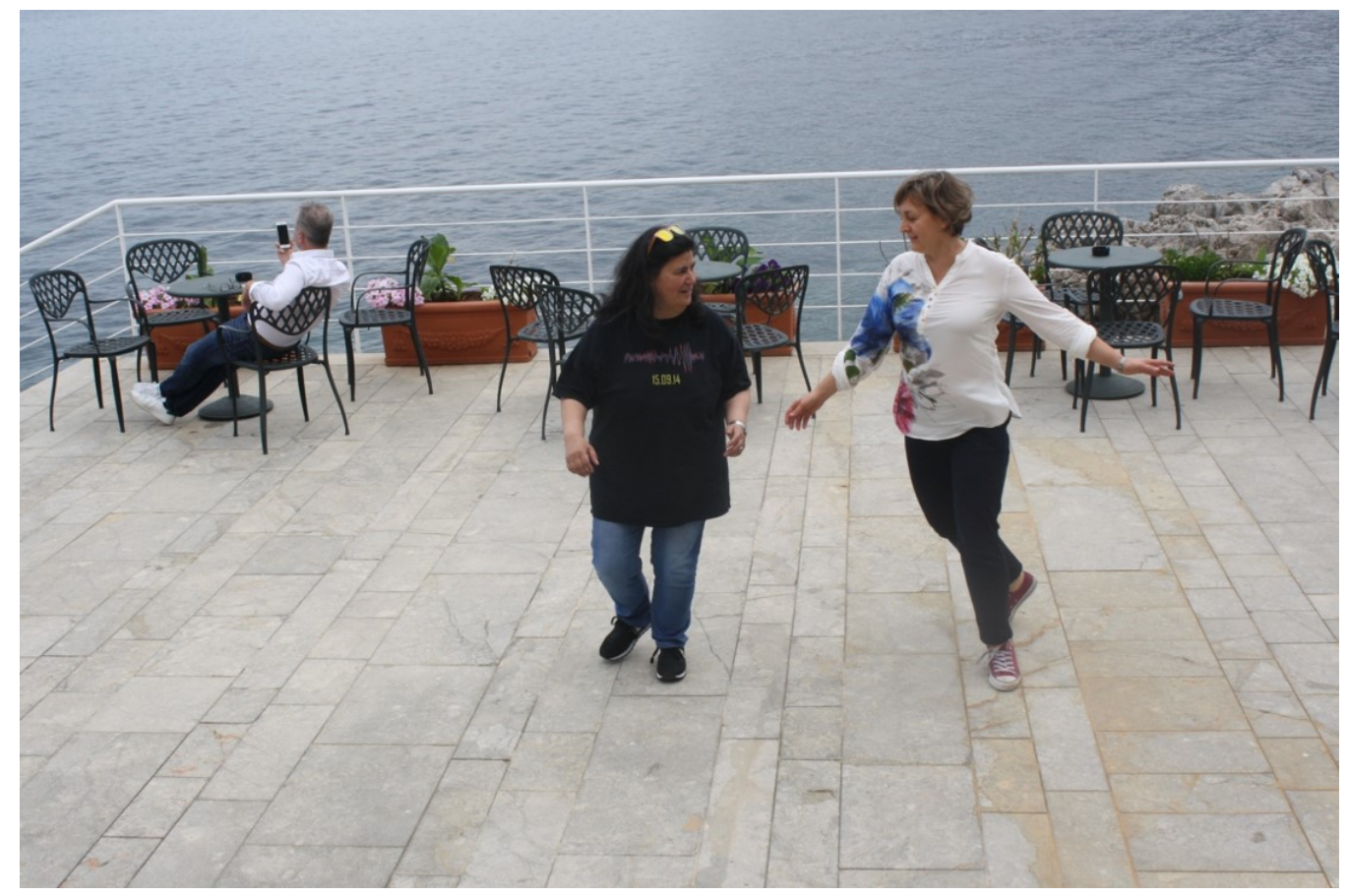

Figure 4: Neutron star - black hole merger by Rosa Poggiani and Dorota Gondek-Rosinska.
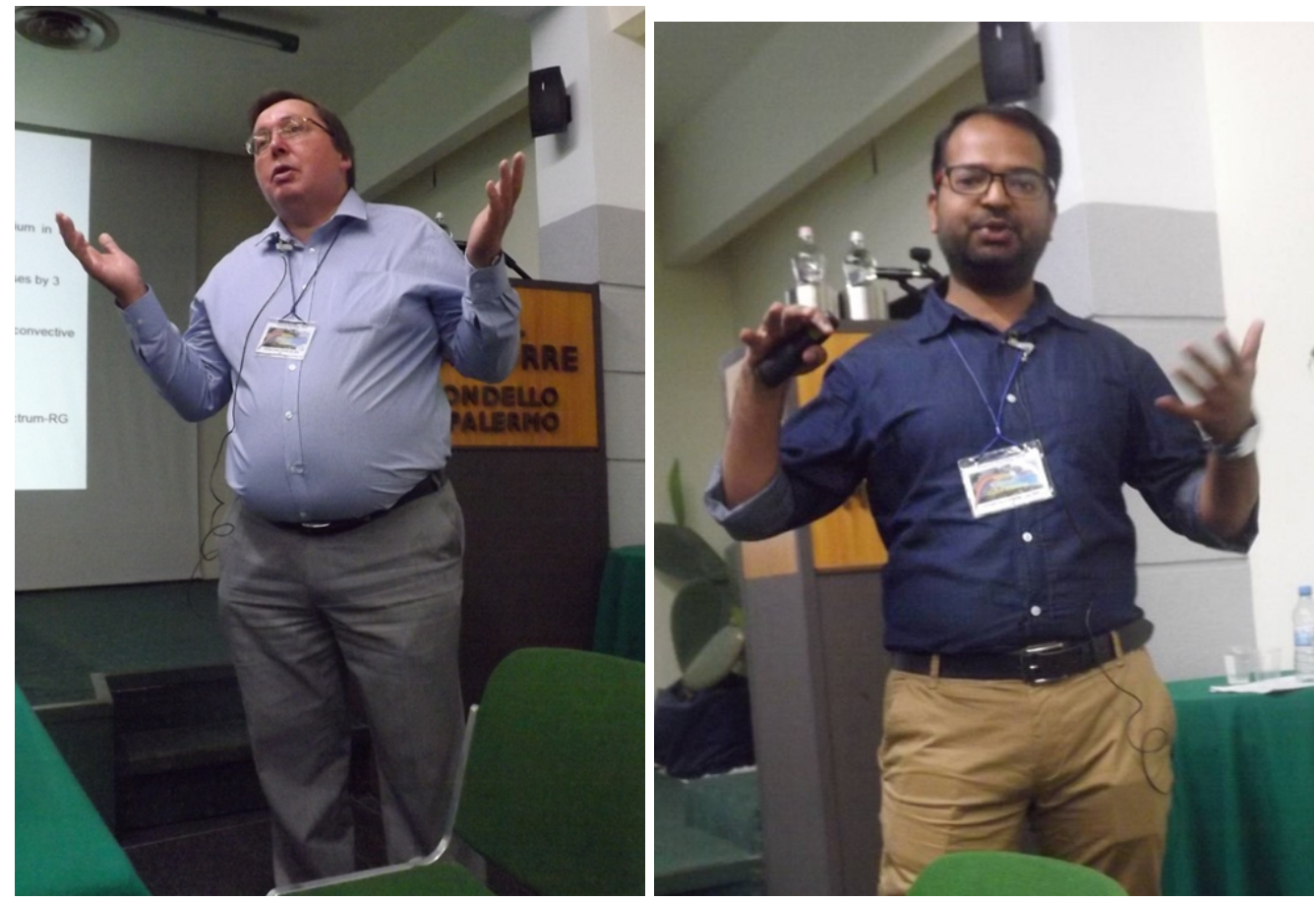

Figure 5: Speakers. 

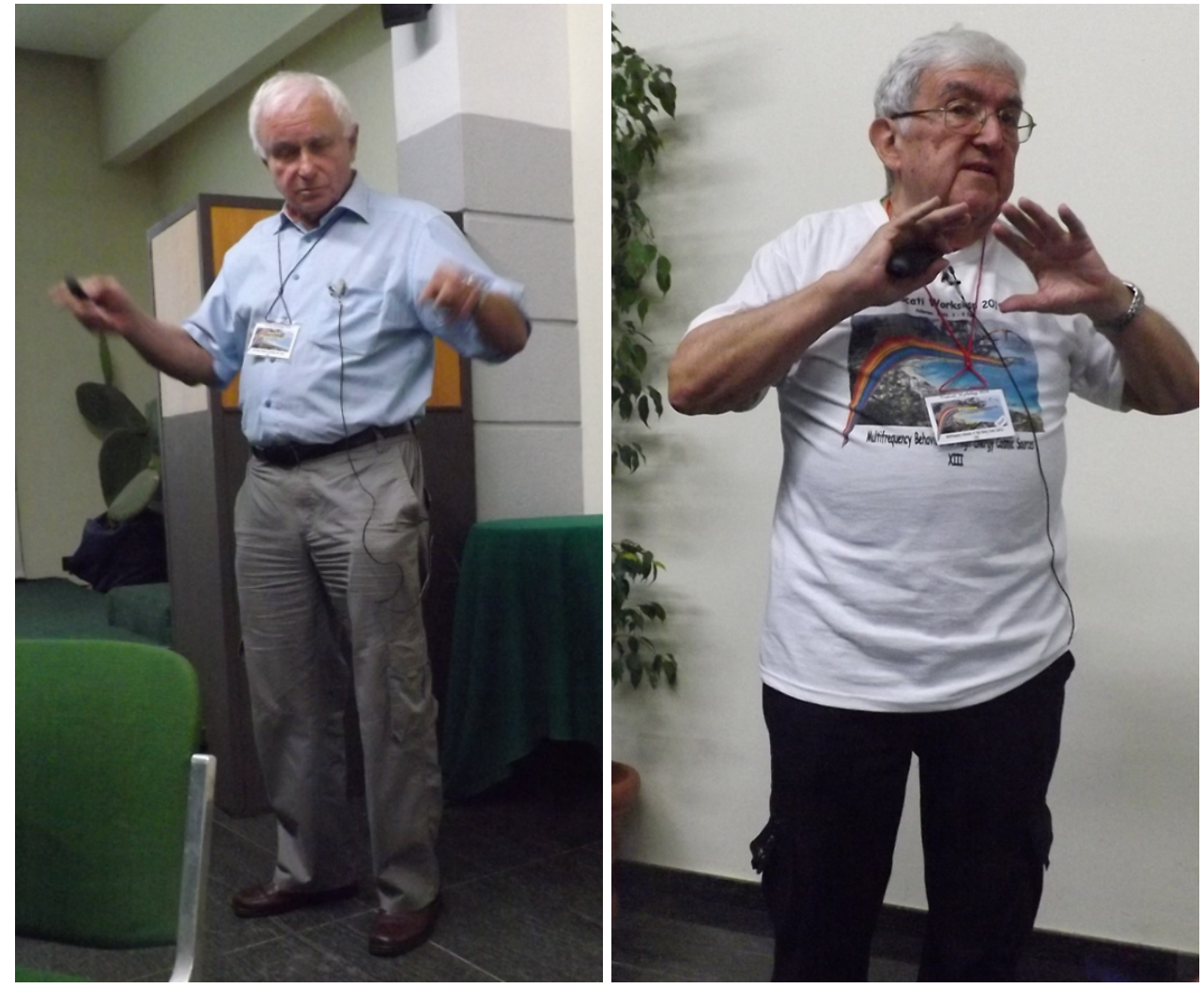

Figure 6: Speakers.
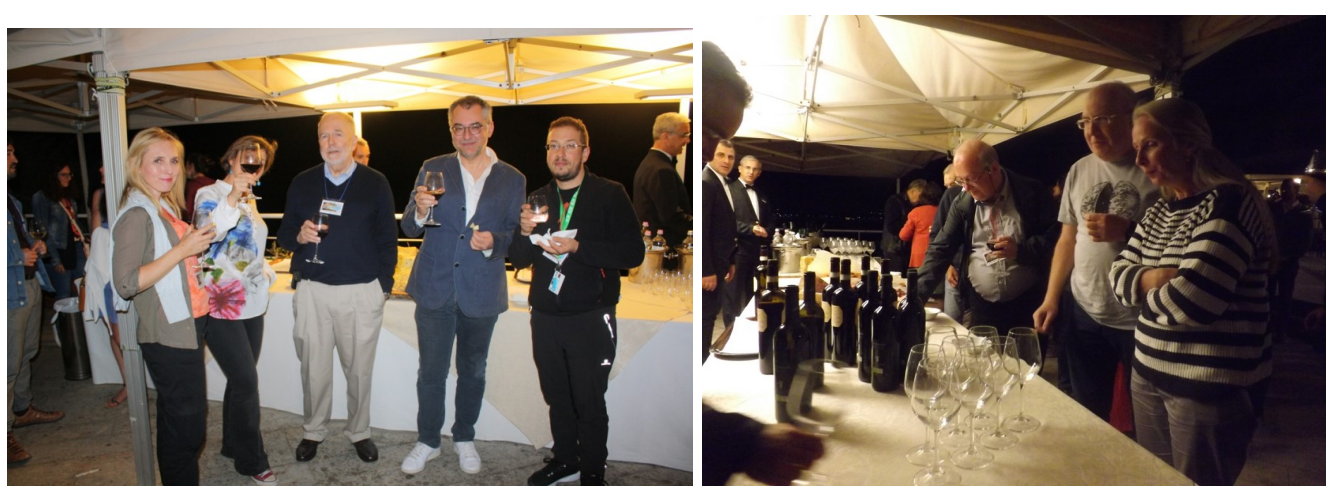

Figure 7: Etruscan wine party.

\section{Conclusions}

In conclusion, I am confident that the goal of the workshop was achieved by the synergy between data from ground-based and space-based experiments as well as results from theoretical work. This work on the forefront of the scientific enterprise is likely to result in high-impact scientific research. Hence, the main purpose of the workshop (namely, to discuss in a unique and collaborative setting a broad range of topics in modern astrophysics) had been achieved. I also believe it will encourage collaborative efforts across various topical areas of research. 


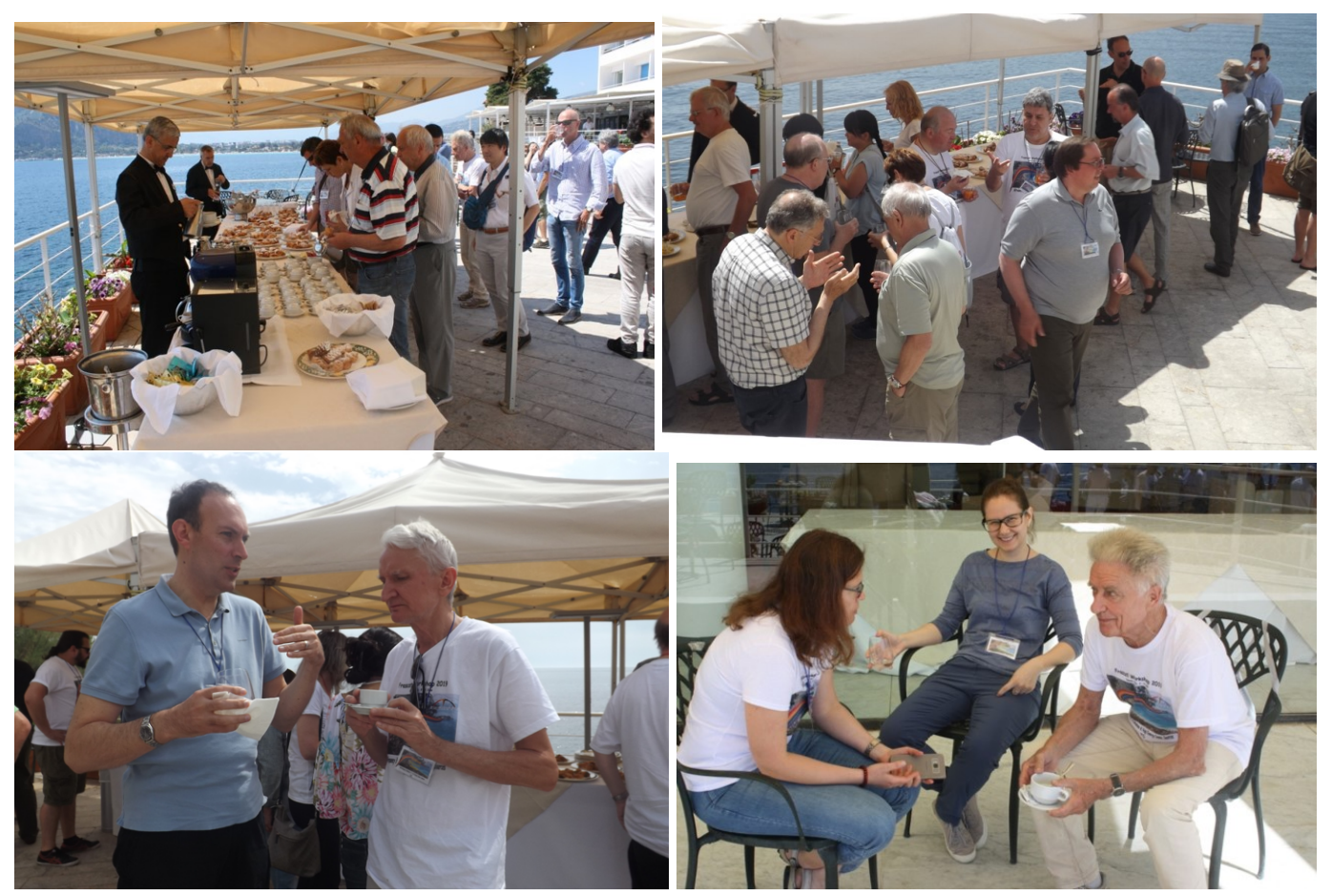

Figure 8: Coffee breaks with delicious Sicilian coffee.

\section{Acknowledgements}

The author acknowledges continuous support by the Astronomical Institute of the Czech Academy of Sciences in Ondrejov under Institutional project RVO 67985815 as well as grants GA CR 13-33324S and Mobility MSMT CZ-A project 7AMB18AT036. The photographs in this paper were provided by Rosa Poggiani and the author. 

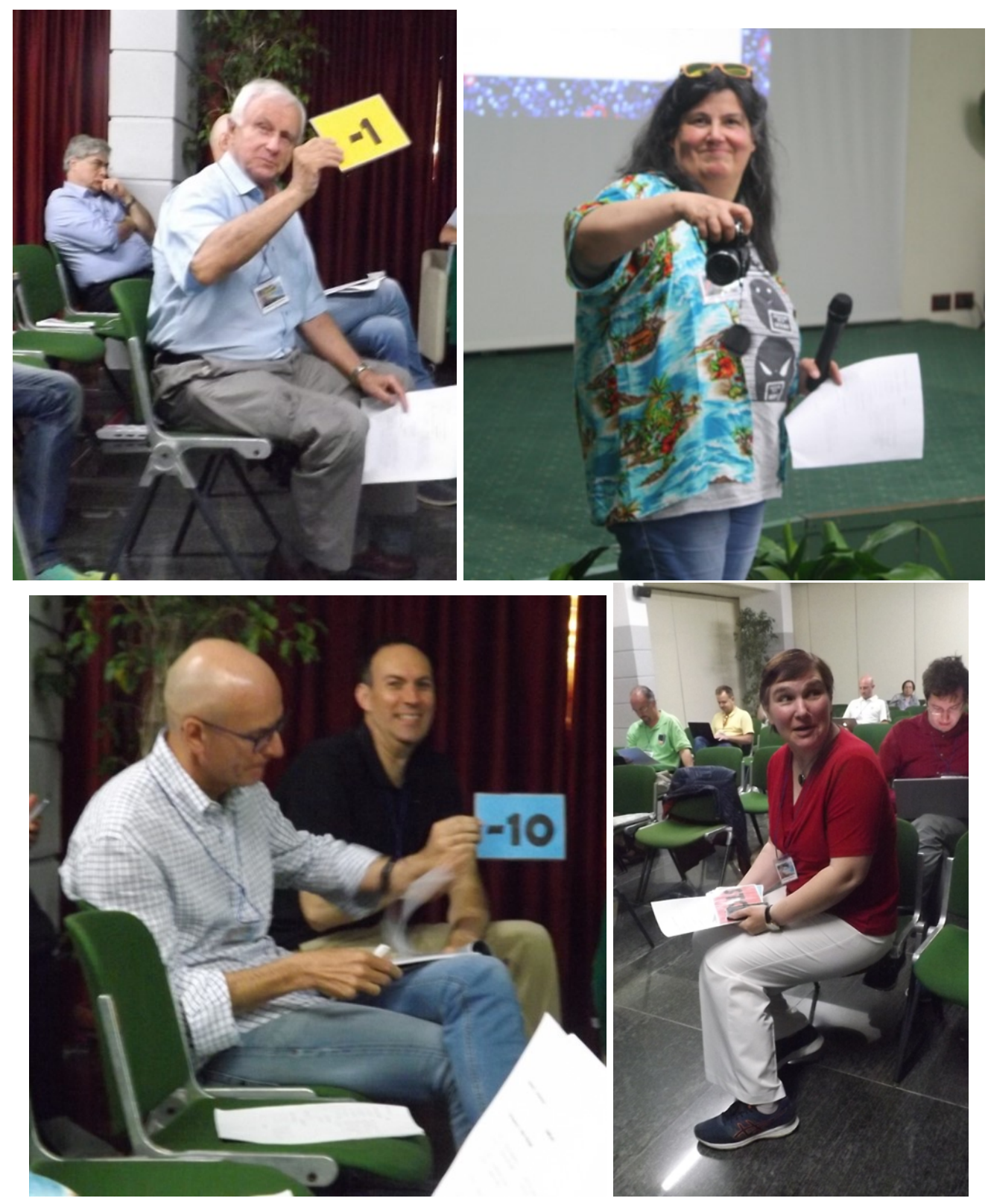

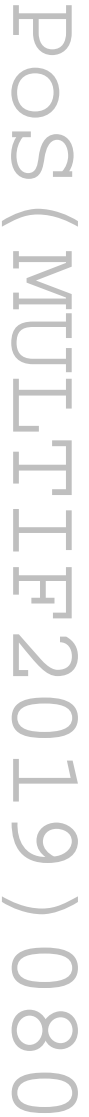

Figure 9: Conference chairs. 


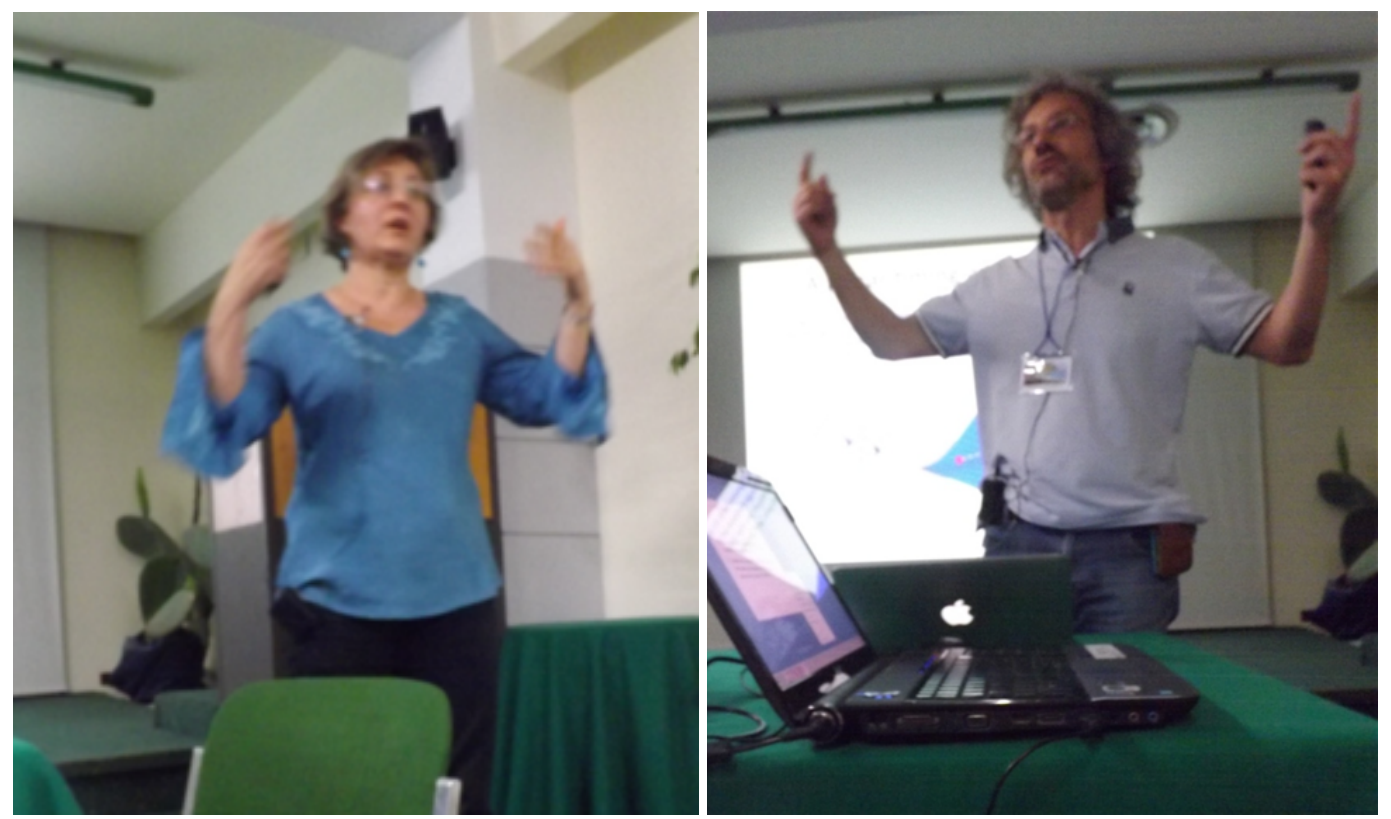

Figure 10: In this conference, quite numerous speakers have illustrated the size of accretion disc in their models, in some cases even in rotation (left).
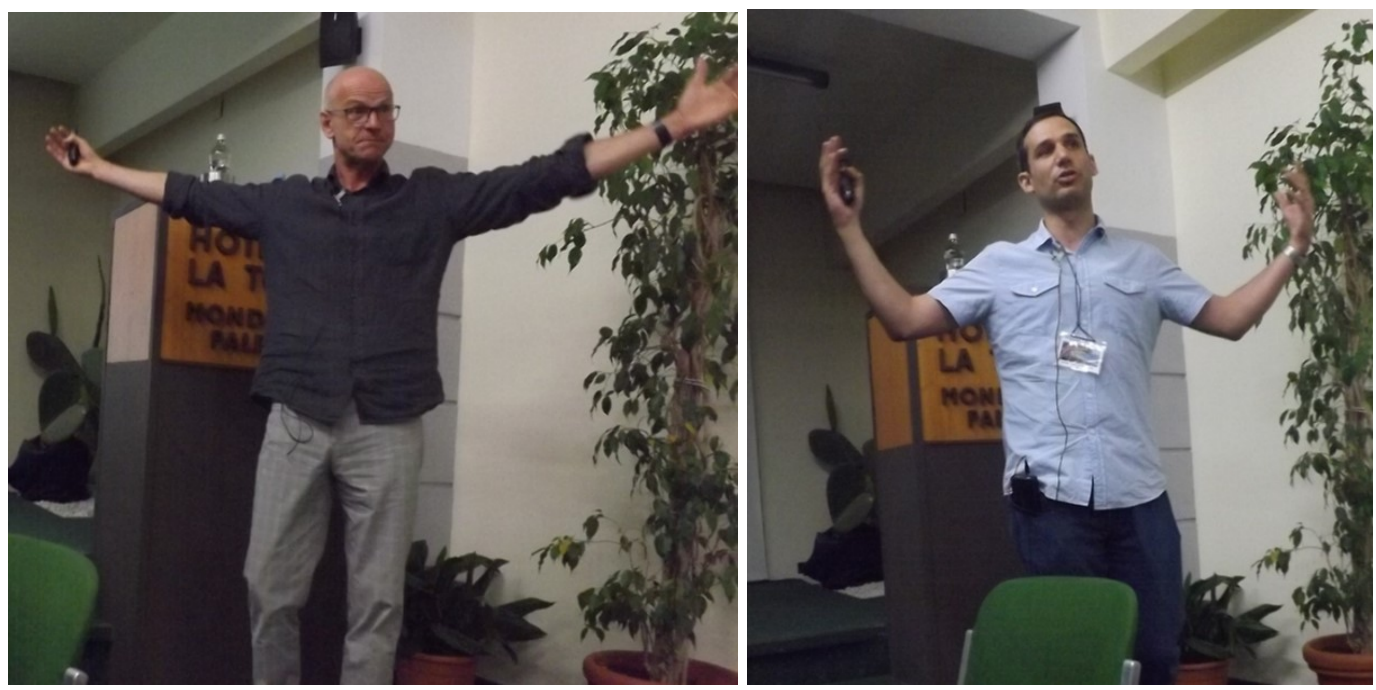

Figure 11: Another speakers demonstrating sizes of their accretion discs... 


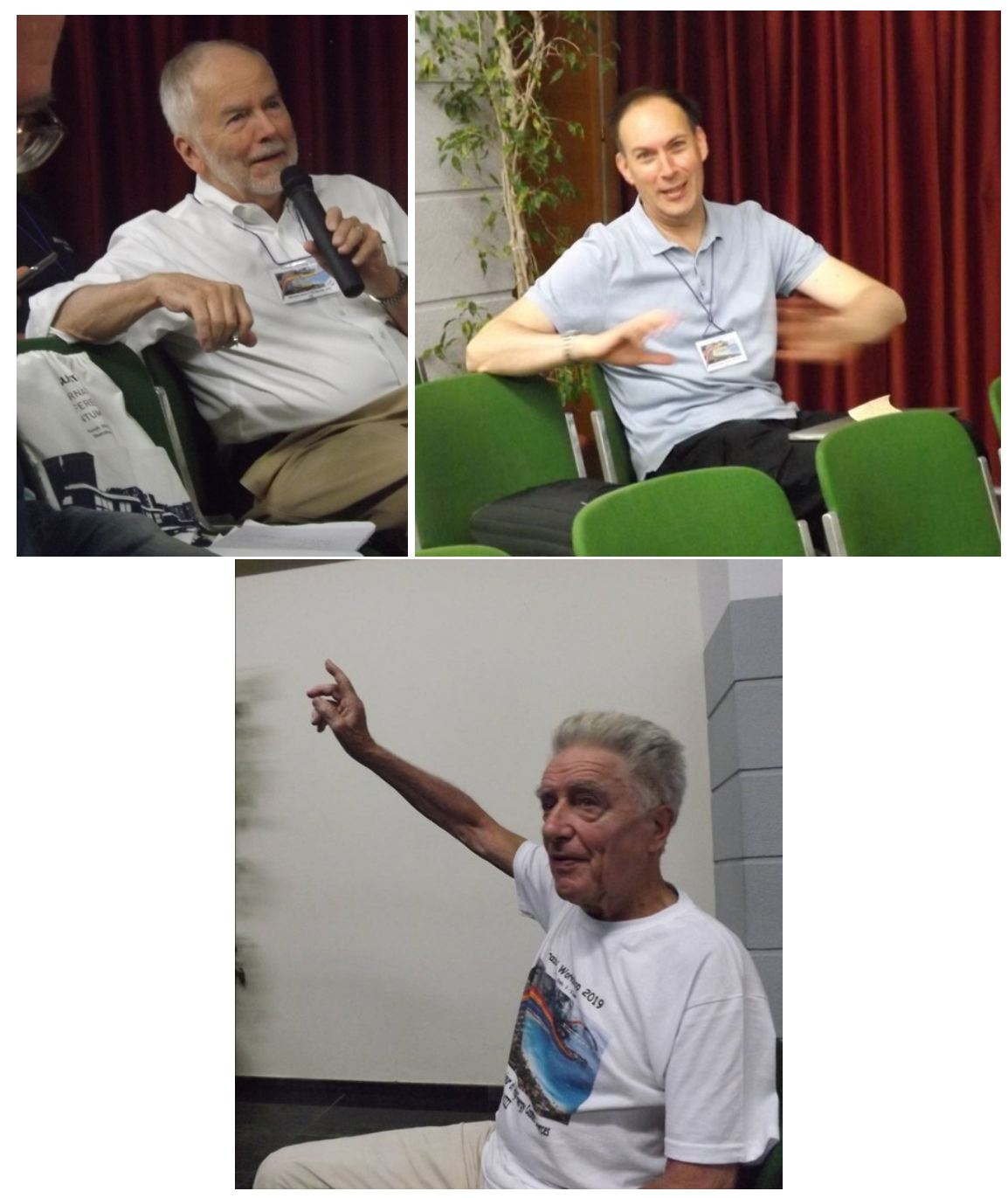

Figure 12: Questions/answers represented important part of the conference. 


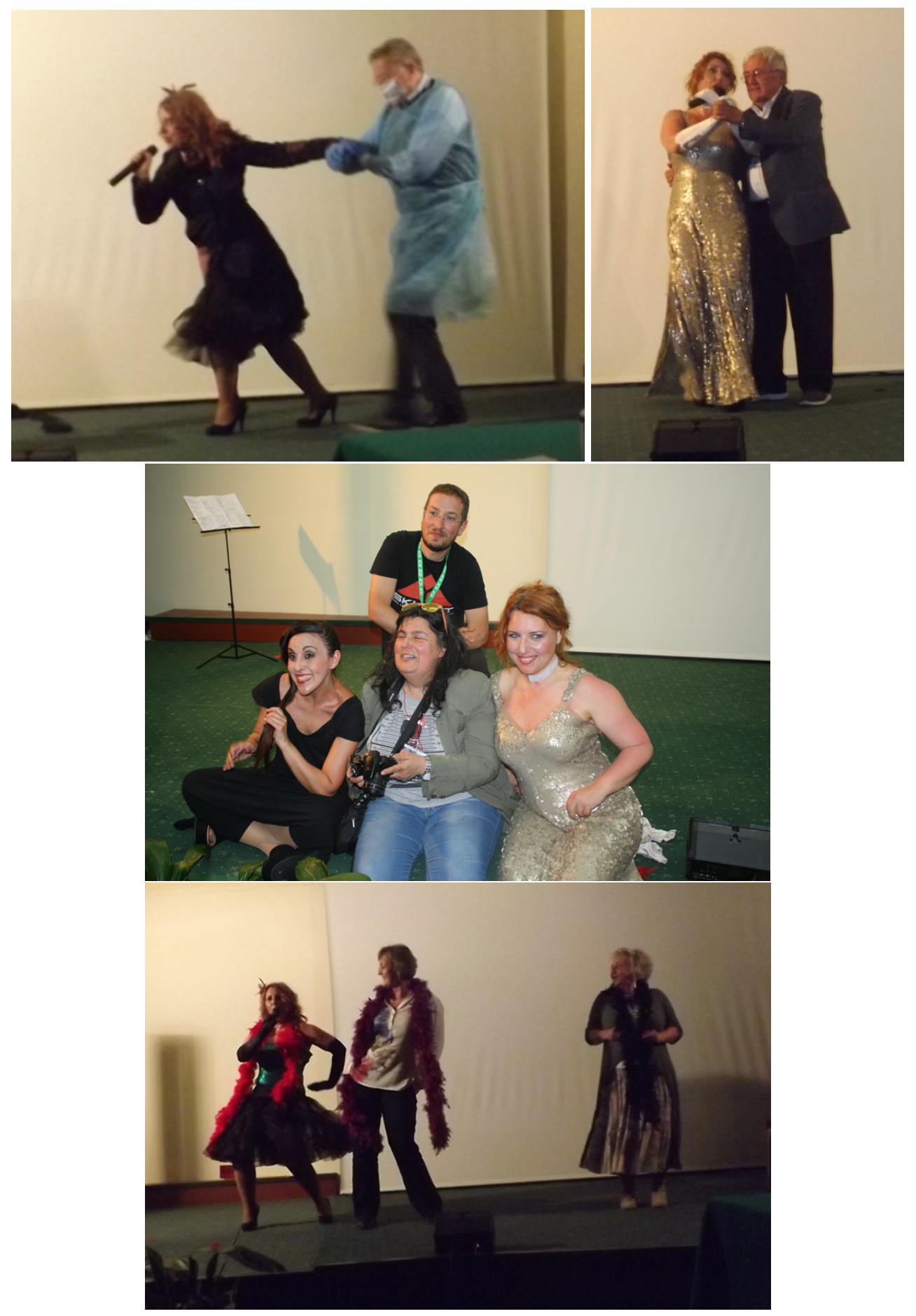

Figure 13: Cultural event. 


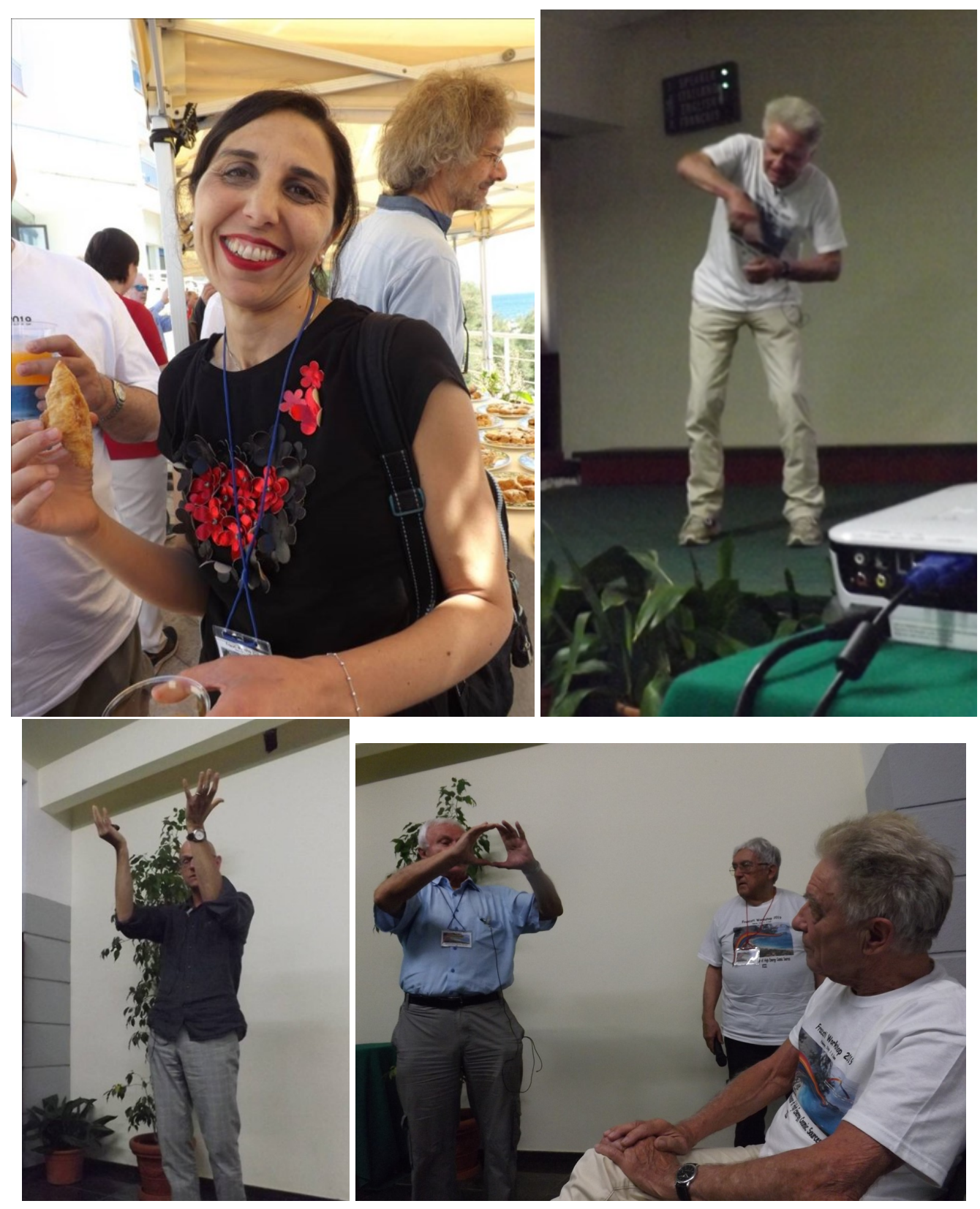

Figure 14: Conference highlights. 

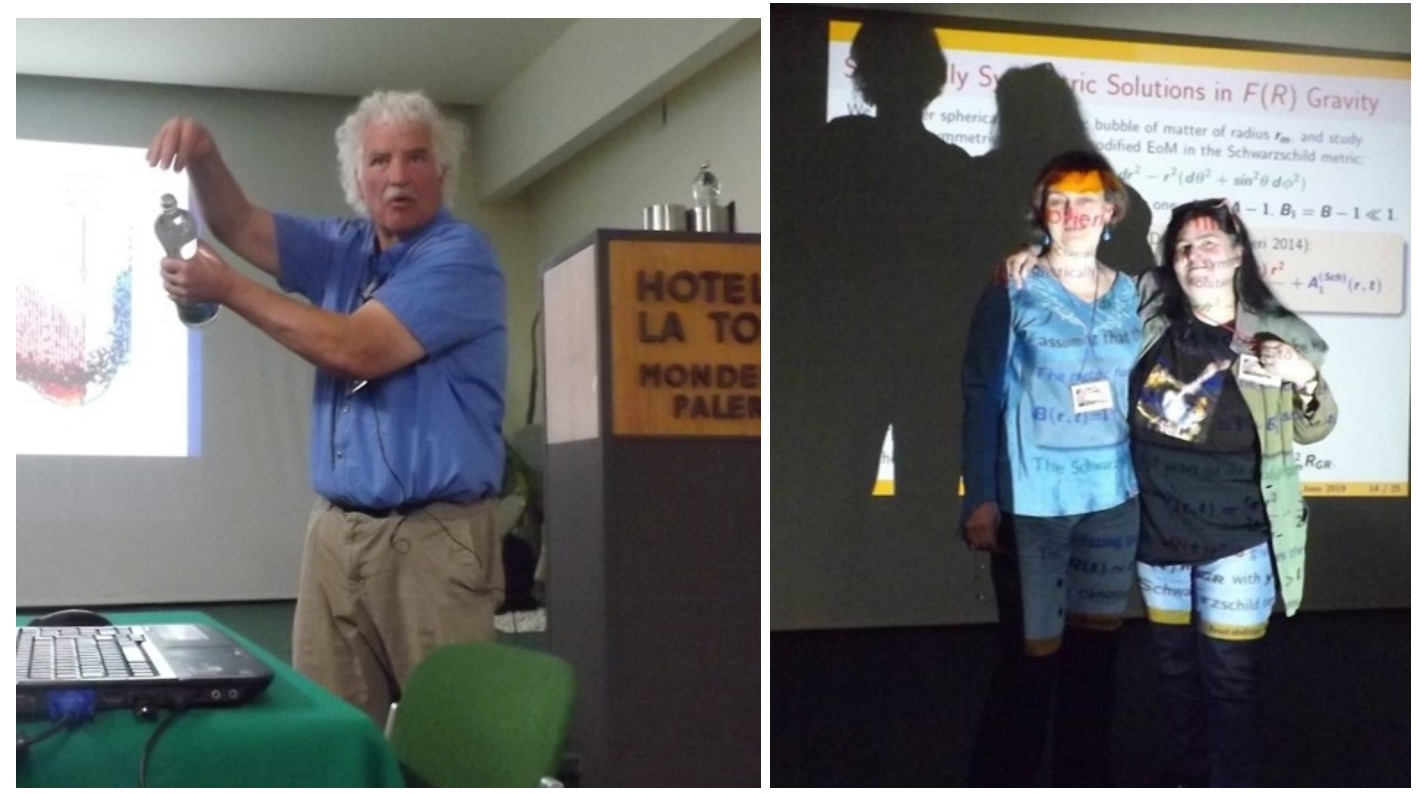

Figure 15: Conference highlights II. 